Mathematical Research Letters 6, 229-236 (1999)

\title{
CONVEX SETS ASSOCIATED WITH VON NEUMANN ALGEBRAS AND CONNES' APPROXIMATE EMBEDDING PROBLEM
}

\author{
FLORIN RĂDULESCU ${ }^{12}$
}

\begin{abstract}
Connes' approximate embedding problem, asks whether any countably generated type $I I_{1}$ factor $M$ can be approximately embedded in the hyperfinite type $I I_{1}$ factor. Solving this problem in the affirmative, amounts to showing that given any integers $N, p$, any elements $x_{1}, \ldots, x_{N}$ in $M$ and any $\epsilon>0$, one can find $k$ and matrices $X_{1}, \ldots, X_{N}$ in the algebra $M_{k}(\mathbb{C})$, endowed with the normalized trace $\mathrm{tr}$, such that for every $i_{1}, \ldots, i_{p} \in\{1, \ldots, N\}$ and for every $s$, with $1 \leq s \leq p$, one has that $\left|\tau\left(x_{i_{1}} \ldots x_{i_{s}}\right)-\operatorname{tr}\left(X_{i_{1}} \ldots X_{i_{s}}\right)\right|<\epsilon$. In this paper we show that this is always possible if $s$ is 2 and 3 .

More precisely we prove that for every strictly positive integer $N$, for every elements $x_{1}, \ldots, x_{N}$ in $M$ and any $\epsilon>0$, one can find $k$ and matrices $X_{1}, \ldots, X_{N}$ in the algebra $M_{k}(\mathbb{C})$, such that for every $i_{1}, i_{2}, i_{3} \in\{1, \ldots, N\}$ one has that $\left|\tau\left(x_{i_{1}} x_{i_{2}} x_{i_{3}}\right)-\operatorname{tr}\left(X_{i_{1}} X_{i_{2}} X_{i_{3}}\right)\right|<\epsilon$ and $\left|\tau\left(x_{i_{1}} x_{i_{2}}\right)-\operatorname{tr}\left(X_{i_{1}} X_{i_{2}}\right)\right|<\epsilon$.

An affirmative solution of the Connes' problem would follow if the above statement could also be proved for $s=4$.
\end{abstract}

\section{Introduction}

In his breakthrough paper on the classification of injective factors $([\mathrm{Co}])$, Connes formulated the question whether any countably generated type $I I_{1}$ factor $M$ can be approximately embedded in the hyperfinite type $I I_{1}$ factor. The recent work of Kirchberg ([Ki]) showed that this question has several equivalent reformulations, one of which is the question whether there exists a unique $C^{*}$ norm on the tensor product of the universal $C^{*}$-algebra of a free group with itself. This problem is also related to Voiculescu's recent work ([Vo]) on modified free entropy. In the paper of Ge and Popa ([GP]) on thin factors, it is proved that any $I I_{1}$ factor may be embedded into a thin factor, hinting that a positive answer should hold for Connes' problem (see also [Po]).

Approximate embedding of a $I I_{1}$ factor $M$ in the hyperfinite type $I I_{1}$ factor is equivalent to showing that for every strictly positive integers $N, p$, for every elements $x_{1}, \ldots, x_{N}$ in $M$ and any $\epsilon>0$, one can find $k$ and matrices $X_{1}, \ldots, X_{N}$

Received October 7, 1998.

1991 Mathematics Subject Classification. Primary 46L35, Secondary 46L37, 46L57.

${ }^{1}$ Research supported in part by the grant DMS 9622911 from the National Science Foundation

${ }^{2}$ Member of the Institute of Mathematics, Romanian Academy, Bucharest 
in the algebra $M_{k}(\mathbb{C})$, endowed with the normalized trace $\operatorname{tr}$, such that for every $i_{1}, \ldots, i_{p} \in\{1, \ldots, N\}$ and for every $s$, with $1 \leq s \leq p$, one has that $\left|\tau\left(x_{i_{1}} \ldots x_{i_{s}}\right)-\operatorname{tr}\left(X_{i_{1}} \ldots X_{i_{s}}\right)\right|<\epsilon$.

In this paper we prove that for any countably generated $I I_{1}$ factor $M$, the following weaker statement holds:

Theorem. For every strictly positive integer $N$, for every element $x_{1}, \ldots, x_{N}$ in $M$, and any $\epsilon>0$, one can find $k$ and matrices $X_{1}, \ldots, X_{N}$ in the algebra $M_{k}(\mathbb{C})$, such that for every $i_{1}, i_{2}, i_{3} \in\{1, \ldots, N\}$, one has that

$$
\left|\tau\left(x_{i_{1}} x_{i_{2}} x_{i_{3}}\right)-\operatorname{tr}\left(X_{i_{1}} X_{i_{2}} X_{i_{3}}\right)\right|<\epsilon
$$

and

$$
\left|\tau\left(x_{i_{1}} x_{i_{2}}\right)-\operatorname{tr}\left(X_{i_{1}} X_{i_{2}}\right)\right|<\epsilon
$$

We also prove that a positive answer to Connes' problem is equivalent to showing that one can choose the matrices $X_{1}, \ldots, X_{N}$, subject to the additional property that the 2-norms $\tau\left(x_{i}^{2} x_{j}^{2}\right)^{1 / 2}$ are also close (within $\epsilon$ ) to the 2-norms $\operatorname{tr}\left(X_{i}^{2} X_{j}^{2}\right)^{1 / 2}$ and such that the uniform norms of $X_{1}, \ldots, X_{N}$ are bounded by a constant, independent of $\epsilon$.

In the proof we consider the closure $K_{M}^{3, N}$ of the set of values of (truncated) noncommutative moments, of order 3 , of $N$ variables in $M$, that are in an orthonormal system. This is a "quantized" version of the Minmax Principle, used to determine the eigenvalues of a compact operator. For factors $M$, whose fundamental group contains a dense multiplicative group, we prove that the set $K_{M}^{3, N}$ is a convex cone. By definition this cone is minimal when $M$ is the hyperfinite factor $R$. To prove the result we will show that the set $K_{R}^{3, N}$ is also maximal. This method is inspired by Voiculescu's definition of free entropy $([\mathrm{Vo}])$.

\section{The results}

Let $p, N$ be arbitrary, strictly positive integers, $p \geq 3$. Let $\mathcal{I}_{N, p}$ be the set of representatives of ordered $s$-tuples, where $3 \leq s \leq p$, of the form $\left(i_{1}, \ldots, i_{s}\right)$, $i_{1}, \ldots, i_{s} \in\{1,2, \ldots, N\}$, subject to the equivalence relation generated by cyclic permutations and reversing the order. If $x_{1}, \ldots, x_{N}$ are variables in an algebra and $I \in \mathcal{I}_{p}$, then we denote by $x_{I}$ the product $x_{i_{1}} \cdots x_{i_{s}}$. Let $r_{p, N}$ be the cardinality of $\mathcal{I}_{N, p}$.

Definition. Let $p, N$ be arbitrary, strictly, positive integers, $p \geq 3$. Let $M$ be a type $I I_{1}$ factor with trace $\tau$. Let $\operatorname{os}_{N}(M)$ be the set of all ordered sets $\left(x_{1}, \ldots, x_{N}\right)$ consisting of selfadjoint elements in $M$, such that $\tau\left(x_{i}^{2}\right)=1$, $\tau\left(x_{i} x_{j}\right)=0$, for all $1 \leq i<j \leq N$.

Let $K_{M}^{N, p}$ be the subset of $\mathbb{C}^{r_{N, p}}$, consisting of the closure of all ordered $r_{N, p^{-}}$ tuples, indexed by $\mathcal{I}_{N, p}$, of moments (of order between 3 and $p$ ) of noncommutative monomials, with variables in orthonormal systems of selfadjoints elements in $M$ : 


$$
K_{M}^{N, p}=\overline{\left\{\left(\tau\left(x_{I}\right)\right)_{I \in \mathcal{I}_{N, p}} \mid\left(x_{1}, x_{2}, \ldots, x_{N}\right) \in o s_{N}(M)\right\}} .
$$

Obviously $K_{M}^{N, p}$ is invariant under the action corresponding to permutation of the elements in $\{1, \ldots, N\}$.

For example $K^{3,3}$ is the closure of the following subset of $\mathbb{C} \times \mathbb{R}^{9}$ :

$$
\begin{gathered}
\left\{\left(\tau(x y z), \tau\left(x^{3}\right), \tau\left(y^{3}\right), \tau\left(z^{3}\right), \tau\left(x^{2} y\right), \tau\left(x^{2} z\right), \tau\left(y^{2} x\right), \tau\left(y^{2} z\right), \tau\left(z^{2} x\right), \tau\left(z^{2} y\right)\right.\right. \\
\left.\mid x, y, z \in M_{\mathrm{sa}}, \tau\left(x^{2}\right)=\tau\left(y^{2}\right)=\tau\left(z^{2}\right)=1, \tau(x y)=\tau(y z)=\tau(x z)=0\right\} .
\end{gathered}
$$

Note that $K^{3,3}$ is a set of ordered pairs. Also note the invariance under transposition which was assumed at the beginning, corresponds to the fact that $\tau(x y z)=\overline{\tau(y x z)}$. Moreover all the other components of elements in $K^{3,3}$ take only real values, since $x, y, z$ are selfadjoint.

The following definition is only used here to get the next proposition in its widest generality.

Definition. Let $L_{M}^{N, p}$ be the set defined by the same requirements as $K_{M}^{N, p}$, except that we replace the set $\mathrm{s}_{N}(M)$, by the set of all $N$-tuples of selfadjoint elements in $M,\left(x_{1}, \ldots, x_{N}\right)$ for which we require only that $\tau\left(x_{i}^{2}\right)=1$.

In the next result we summarize the properties of the sets $K_{M}^{N, p}$ for a general type $I I_{1}$ factor $M$. Recall that for a strictly positive number $t, M_{t}$ is the (unital) isomorphism class of the von Neumann algebras $p(M \otimes B(H)) p$, for any projection $p$ in $M \otimes B(H)$ of trace $t$. The fundamental group of a $I I_{1}$ factor $M$ is the multiplicative subgroup of $\mathbb{R}_{+}$consisting of all strictly positive numbers $t$ such that $M$ is unitarily isomorphic to $M_{t}$ (see $[\mathrm{MvN}]$, [Co], [KaR])

As a corollary we obtain that if $M$ has fundamental group containing a dense subgroup of $\mathbb{R}_{+} \backslash\{0\}$ and $M \cong M \bar{\otimes} M$, then $K_{M}^{N, p}$ is a closed convex set, closed under pointwise multiplication and graded homotethies.

Proposition 1. The sets $L_{M}^{N, p}$ and $K_{M}^{N, p}$ have the following properties

(a) Let $M_{1}, M_{2}$ be two type $I I_{1}$ factors. Denote by $\cdot$ pointwise multiplication on $\mathbb{C}^{r_{N, p}}$. Let $M_{0}=M_{1} \bar{\otimes} M_{2}$. Then for all $N$, $p$ integers with $p \geq 3$ we have $L_{M_{1}}^{N, p} \cdot K_{M_{2}}^{N, p} \subseteq K_{M_{0}}^{N, p}$.

(b) Let $M$ be any type $I I_{1}$ factor and let the $\lambda$ be a number in the interval $(0,1)$. Then, if $\alpha$ belongs to $K_{M_{\lambda}}^{N, p}$ and $\beta$ belongs to $K_{M_{1-\lambda}}^{N, p}$, then $\lambda \alpha+$ $(1-\lambda) \beta$ belongs to $K_{M}^{N, p}$.

(c) Let $M$ be a type $I I_{1}$ factor and let $\lambda$ be any number in the interval $(0,1)$. Let $\Phi_{\lambda}$ be the dilation map on $K_{M}^{N, p}$ multiplying the entries corresponding to monomials of degree $s$, by $\lambda^{1-s / 2}$. Then if $\alpha$ belongs to $K_{M_{\lambda}}^{N, p}$ then $\Phi_{\lambda}(\alpha)$ belongs to $K_{M}^{N, p}$.

(d) Let $\Gamma$ be a discrete, residualy finite group with infinite conjugacy classes. Let $R$ be the hyperfinite $I I_{1}$ factor. Then $K_{R}^{N, p}=K_{\mathcal{L}(\Gamma)}^{N, p}$. Moreover for all type $I I_{1}$ factors $M$ the following holds $K_{R}^{N, p} \subseteq K_{M}^{N, p}$. 
Proof. It is known ([Wa]) that the Connes' conjecture holds for factors $\mathcal{L}(\Gamma)$, where $\Gamma$ is a discrete, residualy finite group with infinite conjugacy classes. This implies $(\mathrm{d})$.

We now prove (c). Assume that $M_{\lambda}$ is realized as $p M p$, where $p$ is a projection in $M$ of trace $\lambda$. Let $\alpha=\left(\tau\left(y_{I}\right)\right)_{I \in \mathcal{I}_{N, p}}$, be an element in $K_{M_{\lambda}, p}^{N, \text { where }}$ $\left(y_{1}, y_{2}, \ldots, y_{N}\right)$ belongs to os $_{N}(p M p)$. Taking into account that the trace on $p M p$ is $(\tau(p))^{-1} \tau$, it follows that $\left(\frac{y_{1}}{(\tau(p))^{1 / 2}}, \ldots, \frac{y_{N}}{(\tau(p))^{1 / 2}}\right)$ belongs to $\operatorname{os}_{N}(M)$. The moments of this new orthonormal system realize $\Phi_{\lambda}(\alpha)$.

The assertion (b) is proved similarly, by a unital embedding of the direct sum of $M_{\lambda} \oplus M_{1-\lambda}$ into $M$. Let $p$ be a projection of trace $\lambda$ in $M$. Let $\left(y_{1}, \ldots, y_{N}\right)=\left(p y_{1} p, \ldots, p y_{N} p\right)$ be an element in $\operatorname{os}_{N}(p M p)$ and let

$$
\left(z_{1}, \ldots, z_{N}\right)=\left((1-p) z_{1}(1-p), \ldots,(1-p) z_{N}(1-p)\right)
$$

be an element in $\operatorname{os}_{N}((1-p) M(1-p))$. We define $x_{i}=y_{i}+z_{i}$. Let $\tau_{p}$ and respectively $\tau_{1-p}$ be the traces on $p M p$ and respectively on $(1-p) M(1-p)$, obtained by renormalizing the trace on $M$. It follows that $\tau\left(y_{i} y_{j}\right)$ is $\tau(p)$ if $i=j$ and zero otherwise. Similarly $\tau\left(z_{i} z_{j}\right)$ is $\tau(1-p)$ if $i=j$ and zero otherwise. Hence $\left(x_{1}, \ldots, x_{N}\right)$ belongs to $\operatorname{os}_{N}(M)$. Moreover for every $I \in \mathcal{I}_{N, p}$, we have that

$\tau\left(x_{I}\right)=\tau\left(y_{I}\right)+\tau\left(z_{I}\right)=\tau(p) \tau_{p}\left(y_{I}\right)+\tau(1-p) \tau_{1-p}\left(z_{I}\right)=\lambda \tau_{p}\left(y_{I}\right)+(1-\lambda) \tau_{1-p}\left(z_{I}\right)$.

The point (a) is proved along the same lines. This completes the proof.

As a corollary of the previous proposition we get the following result:

\section{Corollary 2.}

(a) Let $M$ be a type $I I_{1}$ factor such the fundamental group $\mathcal{F}(M)$ contains the rational numbers. Then $K_{M}^{N, p}$ is convex.

(b) If $M \cong M \bar{\otimes} M$ then $K_{M}^{N, p}$ is closed under pointwise multiplication.

(c) Let $M$ be a type $I I_{1}$ factor such that the fundamental group $\mathcal{F}(M)$ contains the rational numbers. Let $\phi_{i}$, for $i \in\{1, \ldots, N\}$, be the map on $K_{M}^{N, p}$ defined by requiring, for $\alpha \in K_{M}^{N, p}$, that $\phi_{i}(\alpha)$ is the $r_{N, p}$-tuple obtained by replacing by zero all the entries in $\alpha$ corresponding to monomials that involve a total odd power of $x_{i}$. Then $\phi_{i}\left(K_{M}^{N, p}\right)$ is contained in $K_{M}^{N, p}$. The same holds true, for $1 \leq i<j \leq N$, if $\phi_{i, j}$ is the map that in $\alpha$ replaces by zero, all the entries in $\alpha$, corresponding to a total odd power in $x_{i}$ and $x_{j}$.

Proof. (c) is a consequence of (a). The rest is obvious.

Connes' problem has affirmative answer if $K_{M}^{N, p}$ is independent on the choice of $M$ for all $N, p$. In fact, as we will prove bellow, it is sufficient that these statements holds true only for $p=3,4$. It is not clear if the geometry of the set, 
defining $K_{M}^{N, p}$, without considering closure, could be used to distinguish type $I I_{1}$ factors.

The next result concerns the geometry of certain convex sets in $\mathbb{C}^{s} \times \mathbb{R}^{t}$. It will be used to show that $K_{R}^{3, N}$ is maximal.

Lemma 3. Let $K$ be a subset of $\mathbb{C}^{p} \times \mathbb{R}^{q}$ with the following properties:

(i) $K$ is a closed convex cone.

(ii) $K$ is closed under pointwise multiplication.

(iii) For each $s \in\{1, \ldots, q\}, K$ contains two elements of the form $\alpha=\left(z_{1}, \ldots, z_{p}, y_{1}, \ldots, y_{q}\right)$, such that

$$
\left|y_{s}\right|>\max \left\{\left|z_{i}\right|,\left|y_{j}\right| \mid i=1, \ldots, p, j=1, \ldots, q, j \neq s\right\},
$$

and such that $y_{s}$ is positive in one case and negative in the other case.

(iv) For each $r \in\{1, \ldots, p\}, K$ contains an element of the form

$\alpha=\left(z_{1}, \ldots, z_{p}, y_{1}, \ldots, y_{p}\right)$, such that

$$
\left|z_{r}\right|>\max \left\{\left|z_{i}\right|,\left|y_{j}\right| \mid i=1, \ldots, p, j=1, \ldots, q, i \neq r\right\},
$$

and $z_{r}$ is neither purely imaginary and neither real.

Then $K=\mathbb{C}^{p} \times \mathbb{R}^{q}$.

Proof. We will use the following fact: if $z$ is a complex number that is neither real or purely imaginary, then the convex hull of the set $\left\{z^{n} \mid n=1,2, \ldots\right\}$ coincides with $\mathbb{C}$.

Because of property (i) for $K$, we can replace the condition in (iii) by

$$
\left|y_{s}\right|>1>\max \left\{\left|z_{i}\right|,\left|y_{j}\right| \mid i=1, \ldots, p, j=1, \ldots, q, j \neq s\right\},
$$

and similarly for (iv). By using the property (ii) we can show that for each real component $y_{s}$, we can find elements

$$
\alpha=\left(z_{1}, \ldots, z_{p}, y_{1}, \ldots, y_{q}\right),
$$

such that $\left|y_{s}\right|$ is as large as we want, (both positive and negative), while all the other components are as small as we want. Similarly, we can repeat the same procedure for all complex components. The result follows from convexity.

Proposition 4. $K_{R}^{3, N}$ is $\mathbb{R}^{s_{N}} \times \mathbb{C}^{t_{N}}$, where the complex components correspond exactly to those monomials of the form $\tau\left(x_{i} x_{j} x_{k}\right)$, with $i, j, k$ distinct.

Proof. We denote the normalized trace on matrices by tr. By using property (c) from Corollary 4 and because of the previous proposition, the proof is reduced to proving the following assertions:

(A) There exists $(\mathrm{X}, \mathrm{Y}, \mathrm{Z})$ selfadjoint matrices such that $(X, Y, Z)$ is an orthonormal system with respect to the normalized trace and such that $\operatorname{tr}(X Y Z)$ is neither real or purely imaginary, and

$$
\begin{aligned}
|\operatorname{tr}(X Y Z)|>\max \left\{\operatorname{tr}\left(X^{3}\right), \operatorname{tr}\left(Y^{3}\right), \operatorname{tr}\left(Z^{3}\right), \operatorname{tr}\left(X^{2} Y\right), \operatorname{tr}\left(X^{2} Z\right),\right. \\
\left.\operatorname{tr}\left(Y^{2} X\right), \operatorname{tr}\left(Y^{2} Z\right), \operatorname{tr}\left(Z^{2} X\right)\right\} .
\end{aligned}
$$


(B) There exists an orthonormal system $\left(X, T_{1}, \ldots, T_{n}\right)$ in finite matrices, such that

$$
\left|\operatorname{tr}\left(X^{3}\right)\right|>\max \left\{\left|\operatorname{tr}\left(X T_{1}^{2}\right)\right|, \ldots,\left|\operatorname{tr}\left(X T_{n}^{2}\right)\right|\right\} .
$$

(C) There exists an orthonormal system $\left(X, Y, T_{1}, \ldots, T_{n}\right)$ in finite matrices such that

$$
\left|\operatorname{tr}\left(X^{2} Y\right)\right|>\max \left\{\left|\operatorname{tr}\left(Y^{3}\right)\right|,\left|\operatorname{tr}\left(T_{1}^{2} Y\right)\right|, \ldots,\left|\operatorname{tr}\left(T_{n}^{2} Y\right)\right|\right\} .
$$

Observe that by applying the symmetry which replaces simultaneously $X, Y$ by $-X,-Y$, and then using convexity, it follows that (A) is reduced to finding $(\mathrm{X}, \mathrm{Y}, \mathrm{Z})$ selfadjoint matrices (or elements in $R$ ) such that $(X, Y, Z)$ is an orthonormal system, $\operatorname{tr}(X Y Z)$ is neither real or purely imaginary, and

$$
\left(A^{\prime}\right)|\operatorname{tr}(X Y Z)|>\max \left\{\operatorname{tr}\left(Z^{3}\right), \operatorname{tr}\left(X^{2} Z\right), \operatorname{tr}\left(Y^{2} Z\right)\right\} .
$$

By applying the symmetry which replaces simultaneously $Z, Y$ by $-Z,-Y$, and then using convexity, it follows that $\left(A^{\prime}\right)$ is reduced to finding $(\mathrm{X}, \mathrm{Y}, \mathrm{Z})$ selfadjoint matrices (or elements in $R$ ) such that $(X, Y, Z)$ is an orthonormal system, $\operatorname{tr}(X Y Z)$ is neither real or purely imaginary, and

$$
\left(A^{\prime \prime}\right) \quad|\operatorname{tr}(X Y Z)|>0 .
$$

This is obvious.

To find examples as in (B), (C) it is sufficient to find the corresponding elements in the commutative algebra of a probability space (which is always a subalgebra of the hyperfinite factor).

Point (B) can be solved by requiring that $T_{1}, \ldots, T_{n}$ are independent of $X$, Then (B) is equivalent to finding $X$ such that $\left|\operatorname{tr}\left(X^{3}\right)\right|>|\operatorname{tr}(X)|$.

To prove $(\mathrm{C})$ we also require that $T_{1}, T_{2}, \ldots, T_{n}$ are in an algebra that is independent to the algebra generated by $X, Y$. Then it remains to find $X, Y$ an orthonormal system such that

$$
\left|\operatorname{tr}\left(X^{2} Y\right)\right|>\max \left\{\left|\operatorname{tr}\left(Y^{3}\right)\right|,|\operatorname{tr}(Y)|\right\} .
$$

By requiring that $Y$ and $Y^{3}$ have zero expectation, while $\left|\operatorname{tr}\left(X^{2} Y\right)\right| \neq 0$ the result follows.

This completes the proof.

Theorem. Let $M$ be any type $I I_{1}$ factor with trace $\tau$. Then for every strictly positive integer $N$ and for every set of elements $x_{1}, \ldots, x_{N} \in M$ and for any $\epsilon>0$, there exists matrices $X_{1}, X_{2}, \ldots, X_{N}$ of sufficiently large size, such that if $\operatorname{tr}$ is the normalized trace on matrices, then for all $i, j, k \in\{1,2, \ldots, N\}$ we have that

$$
\left|\tau\left(x_{i} x_{j} x_{k}\right)-\operatorname{tr}\left(X_{i} X_{j} X_{k}\right)\right|<\epsilon, \quad\left|\tau\left(x_{i} x_{j}\right)-\operatorname{tr}\left(X_{i} X_{j}\right)\right|<\epsilon .
$$

Proof. For the proof we can assume that $x_{1}, \ldots, x_{N}$ are an orthonormal system consisting of selfadjoint elements. By the previous proposition $K_{M}^{3, N}$ is independent of the choice of the factor $R$ and hence its equal to $K_{R}^{3, N}$. This completes the proof. 
Remark. Assume that $M$ is a type $I I_{1}$ factor such for any set of selfadjoint elements $x_{1}, \ldots, x_{N} \in M$ there exists a constant $C$ such that for any $\epsilon>0$, there exist selfadjoint matrices $X_{1}, X_{2}, \ldots, X_{N}$ of of uniform norm less than $C$ with the following properties: for all $i, j, k \in\{1,2, \ldots, N\}$ we have that

$$
\left|\tau\left(x_{i} x_{j} x_{k}\right)-\operatorname{tr}\left(X_{i} X_{j} X_{k}\right)\right|<\epsilon,\left|\tau\left(x_{i} x_{j}\right)-\operatorname{tr}\left(X_{i} X_{j}\right)\right|<\epsilon,
$$

and in addition,

$$
\left|\tau\left(x_{i}^{2} x_{j}^{2}\right)-\operatorname{tr}\left(X_{i}^{2} X_{j}^{2}\right)\right|<\epsilon .
$$

Then, for every integer $p \geq 1$, for any set of elements $x_{1}, \ldots, x_{N} \in M$ and for any $\epsilon>0$, there exists matrices $X_{1}, X_{2}, \ldots, X_{N}$ such that for all $i_{1}, i_{2}, \ldots, i_{p} \in\{1,2, \ldots, N\}$ and for all $s=1,2, \ldots, p$, we have that

$$
\left|\tau\left(x_{i_{1}} \cdots x_{i_{s}}\right)-\operatorname{tr}\left(X_{i_{1}} \cdots X_{i_{s}}\right)\right|<\epsilon
$$

Proof. By eventually replacing the set $x_{1}, x_{2}, \ldots, x_{N}$ with a larger set such that every element in the older set is a product of three elements in the newer set, we can reduce the proof to the case when $s \geq 3$. For any $\epsilon>0$, we apply the hypothesis for a much smaller $\epsilon^{\prime}$ and for the set of all $x_{I}, I \in \mathcal{I}_{2 p, N}$. In this way we get a family of matrices $\left(X_{I}\right)_{I \in \mathcal{I}_{2 p, N}}$. By $I \# J$ we denote the concatenation of two elements in $\mathcal{I}_{p, N}$.

The hypothesis shows that for all $I, J \in \mathcal{I}_{p, N}$ and all $K \in \mathcal{I}_{2 p, N}$ we have that

$$
\left.\left.\mid \operatorname{tr}\left(X_{I \# J} X_{K}\right)-\tau\left(x_{I \# J} x_{K}\right)\right) \mid<\epsilon^{\prime} \text { and } \mid \operatorname{tr}\left(X_{I} X_{J} X_{K}\right)-\tau\left(x_{I} x_{J} x_{K}\right)\right) \mid<\epsilon^{\prime} .
$$

Hence, for all $K \in \mathcal{I}_{2 p, N}$ we have that

$$
\left.\mid \operatorname{tr}\left(X_{I \# J} X_{K}\right)-\operatorname{tr}\left(X_{I} X_{J} X_{K}\right)\right) \mid<3 \epsilon^{\prime} .
$$

Hence the projection of $X_{I} X_{J}$ onto the subspace generated by all the $X_{K}, K \in$ $\mathcal{I}_{2 p, N}$, is close to $X_{I \# J}$. The fact that $X_{I} X_{J}$ and $X_{I \# J}$ have also close norms implies that $X_{I} X_{J}$ is close (depending on a function of $\epsilon$ ) to $X_{I \# J}$. Hence when estimating traces of products of more than three elements (by eventually decreasing the order of approximation), we may regroup some of the terms, and hence use the approximations valid for the trace of a product of three elements.

\section{References}

[Co] A. Connes, Classification of injective factors, Cases $I I_{1}, I I_{\infty}, I I I_{\lambda}, \lambda \neq 1$, Ann. of Math. (2) 104 (1976), 73-115.

[GP] L. Ge and S. Popa, On some decomposition properties for factors of type $I I_{1}$, preprint 1997.

[KaR] R.V. Kadison and J.R. Ringrose, Fundamentals of the theory of operator algebra, Pure and Applied Mathematics, 100., vol. I, II, Academic Press, New York-London, 1983. 
[Ki] E. Kirchberg, On nonsemisplit extensions, tensor products and exactness of group C*algebras 112 (1993), Invent. Math., 449-489.

[MvN] F.J. Murray and J. von Neumann, On ring of Operators. IV., Annals of Math. (2) 44 (1943), 716-808.

[Pi] G. Pisier, A simple proof of a theorem of Kirchberg and related results on $C *$-norms, J. Operator Theory 35 (1996), 317-335.

[Po] S. Popa, Free independent sequences in type II $I_{1}$ factors, Proceedings of the Operator Algebras Conference 1992, Orléans (G. Skandalis, ed.), Paris, France, June 1995.

[Wa] S. Wassermann, Exact $C^{*}$-algebras and related topics, Lecture Notes Series, 19., Seoul National University, Research Institute of Mathematics, Global Analysis Research Center, Seoul, 1994.

[Vo] D. Voiculescu, A strengthened asymptotic freeness result for random matrices with applications to free entropy, Internat. Math. Res. Notices 1 (1998), 41-63.

Department of Mathematics, The University of Iowa, Iowa City, IA 52246

E-mail address: radulesc@math.uiowa.edu 